A Multidisciplinary Publication of Centre for Research,

Maitreyi College, University of Delhi

April 2020, Volume 1, Issue 1

Original Research Article

\title{
Influence of the Homeopathic Medicine, Arsenicum album, on Selected Biochemical Parameters of Avian and Mammalian Blood in vitro
}

\author{
Meena Yadav*, Mukta Yadav and Lavanya Ranjan \\ Department of Zoology, Maitreyi College, University of Delhi, New Delhi- 110021 \\ *Correspondence: drmeena.yadav@gmail.com
}

\begin{abstract}
Spectroscopy has been established as the biological tool for diagnosis of serum to know the health of a person. In our study, we incubated chicken and goat blood with five doses (4\%, 2\%, 1.34\%, $1 \%$ and $0.5 \%)$ of homeopathic medicine Arsenicum album-30C and their respective controls. The optical densities of the blood were measured and compared. In chicken blood, there was an increase in the amino acid levels, glucose metabolism and carboxyhemoglobin levels with 1.34\%, $1 \%$ and $0.5 \%$ Arsenicum album as compared to control. Further, 1.34\% Arsenicum album showed maximum therapeutic effects as it lowered protein breakdown, carbohydrate metabolism and oxidative stress and also lowered the levels of carboxyhemoglobin and oxyhemoglobin after 30 minutes of incubation. With $1 \%$ Arsenicum album, there was reduced cellular breakdown, lowered carbohydrate metabolism and hemoglobin degradation after 30 minutes of incubation. Incubation of goat blood with $1.34 \%$ and 1\% Arsenicum album increased the levels of carboxyhemoglobin but decreased the levels of amino acid and glucose metabolism. Arsenicum album showed healing effects, in goat blood, with only two doses i.e. $2 \%$ and $1 \%$. After incubation with $2 \%$ and $1 \%$ Arsenicum album, the cellular breakdown was significantly lowered after 30 minutes and 60 minutes of incubation while the other doses of Arsenicum album did not show any therapeutic effects on the cellular components of blood but in fact induced deleterious effects.
\end{abstract}

Keywords: Arsenicum album 30C, carboxyhemoglobin, oxyhemoglobin, erythrocytes, optical density (OD)

\section{INTRODUCTION}

The branch of homeopathy was propounded by Samuel Hahnemann in 1796 and it was created as an alternate medicine as at that time popular medicines were accompanied by several adverse effects. It can also be said that homeopathy is one of the most controversial forms of complementary and alternative medicine (Caulfield et al., 2005). Homeopathic medicines have been used to cure several diseases like respiratory tract infections, depression and even AIDS 
(Beghi et al., 2016 \& Vilsveen et al., 2018). Treatment of an ailment through homeopathic medicines is based on the law of similar and the current principle working at the heart of homeopathy is 'Similia Similibus Curentur' viz. 'like cures like'. It is believed that if a particular medicine induces certain symptoms in a healthy individual, then it also possesses the ability to cure the same symptoms in a diseased person (Loudon, 2006).

Arsenicum album, a common homeopathic medicine, is generally prescribed by the homeopaths for various symptoms like cough, cold, fever, insomnia, digestive disorders due to intake of cold food or drinks, allergies, depression, anxiety and leukaemia to name a few. It has been shown that people who live in high risk arsenic contaminated areas show improvements in their health like ameliorated levels of liver enzymes and skin symptoms when administered Arsenicum album (Khuda-Baksh et al., 2005). Arsenicum album is commonly used in eastern India and Bangladesh, as a remedy of arsenic poisoning caused from the drinking water (Ive et al., 2012). It is believed that homeopathic drugs stimulate a person's mind into believing that he/she is being healed which eventually leads to the immune system becoming activated. Critics have argued endlessly, about homeopathy being nothing more than a "placebo" and having no real medicinal consequences' (Antonelli \& Donelli, 2019).

The understanding about the underlying mechanisms of action of homeopathic drugs is still in its infancy. Although homeopathy is a well-known form of treatment among people in general, it is still not acknowledged by doctors and/or academicians (Steuter, 2002). The problem with the acceptance of the homeopathic medicines is the lack of clinical and evidence-based studies. Cell-based studies can be used to study the functions of homeopathic drugs (Ive et al., 2012). However, lately doctors have started to acknowledge the potential of homeopathy and have started to use it in their clinical practices in the form of an integration to their existing practice (Bellavite, 2015). In the light of the scant available information on the effects of homeopathic drugs on cellular components of blood, the study was designed to examine the effects of Arsenicum album, a commonly used homeopathic drug, on the biochemical parameters of mammalian blood in vitro.

\section{MATERIAL AND METHODS}

The homeopathic medicine Arsenicum album of 30C potency, on lactose pellet, was used for the experiment. Fresh goat and chicken blood were collected early morning on the day of the experiment, from a butcher house in Satyaniketan, South Delhi, in EDTA containing tarson tubes. Blood was diluted to 1:100 with phosphate buffered saline (PBS) (pH 7.4). Five different 
concentrations $(4 \%, 2 \%, 1.34 \%, 1 \% \& 0.5 \%)$ of lactose pellets of Arsenicum album were prepared in PBS. Similarly, five different concentrations of plain lactose pellets were prepared in PBS to act as controls.

The changes in the biochemical parameters of blood due to Arsenicum album were assessed as per the method standardized by Yadav et al., (2020). Diluted goat and chicken blood were incubated with five different concentrations of Arsenicum album and control, in the ratio of 1:100, for various time intervals viz. $0 \mathrm{~min}, 30 \mathrm{~min}$ and $60 \mathrm{~min}$. The blood was then centrifuged after incubation and supernatant was isolated. Optical densities (OD) of the supernatants were measured at different wavelengths ( $\lambda$ ) viz. $280 \mathrm{~nm}$ (amino acids), $346 \mathrm{~nm}$ (NADH \& NADPH), $418 \mathrm{~nm}, 544 \mathrm{~nm}$ and $578 \mathrm{~nm}$ (carboxyhemoglobin and oxyhemoglobin) in UV- VIS spectrophotometer. All the experiments were performed in triplicates.

\subsection{Statistical Analysis}

The ODs are expressed as Mean (Standard Deviation) for all the samples studied. The data was also subjected to paired sample t-test to calculate the significance of the difference between the OD values at three time intervals viz. just after incubation, 30 minutes after incubation and 60 minutes after incubation with $95 \%$ CI. A p value of $\leq 0.05$ is considered statistically significant. The analysis was done using statistical software SPSS version 20.0.

\section{RESULTS}

After incubation of chicken blood with selected strengths of Arsenicum album, it was observed that in general the OD values were lower in experimental groups $(4 \%, 2 \%, 1.345,1 \%$ and $0.5 \%$ ) as compared to control but after incubation with $1.34 \%$ medicine, OD values increased at $418 \mathrm{~nm}$ and $544 \mathrm{~nm}$ just after incubation; with $1 \%$ medicine incubation OD values increased at $346 \mathrm{~nm}$ and $418 \mathrm{~nm}$ and with $0.5 \%$ medicine, the OD values increased sharply at $418 \mathrm{~nm}$. Further, with $4 \%, 2 \%$ and $0.5 \%$ medicine incubation, the OD values increased after 30 minutes of incubation at $418 \mathrm{~nm}$ while with $4 \%, 2 \%$ and $0.5 \%$ placebo incubation, the OD values decreased at $418 \mathrm{~nm}$ (Table 1).

When the goat blood was incubated with various strengths $(4 \%, 2 \%, 1.34 \%, 1 \%$ and $0.5 \%)$ of Arsenicum album, the OD values did not show much variation as compared to control. However, OD increased significantly at $280 \mathrm{~nm}, 346 \mathrm{~nm}$ and $418 \mathrm{~nm}$ after 30 minutes of incubation with $1.34 \%$ medicine. After incubation with $1 \%$ medicine the OD values increased 
at $280 \mathrm{~nm}, 346$ and $418 \mathrm{~nm}$ just after incubation and at $280 \mathrm{~nm}$ and $346 \mathrm{n}$ after 30 minutes of incubation as compared to control (Table 2).

Table 1: Changes in the optical density of blood after incubation with Arsenicum album of various strengths with chicken blood at selected wavelengths at two time intervals viz. just after incubation and 30 minutes after incubation [Values are expressed as Mean(SD)]

\begin{tabular}{|c|c|c|c|c|c|c|c|c|}
\hline \multirow{2}{*}{$\begin{array}{c}\lambda \\
(\mathbf{n m})\end{array}$} & \multicolumn{2}{|c|}{ Control (2\%) } & \multicolumn{2}{|c|}{ Experimental (2\%) } & \multicolumn{2}{|c|}{ Control $(1.34 \%)$} & \multicolumn{2}{|c|}{ Experimental (1.34\%) } \\
\hline & 0 min & $30 \mathrm{~min}$ & $0 \mathrm{~min}$ & $30 \mathrm{~min}$ & 0 min & $30 \mathrm{~min}$ & 0 min & $30 \mathrm{~min}$ \\
\hline 280 & $0.261(0.0043)$ & $0.255(00015)$ & $0.235(0.0021)$ & $0.248(0.003)$ & $0.370(0.010)$ & $0.343(0.002)$ & $0.391(0.006)$ & $0.344(0.002)$ \\
\hline 346 & $0.052(0.0012)$ & $0.047(0.0025)$ & $0.035(0.0038)$ & $0.043(0.0026)$ & $0.080(0.002)$ & $0.060(0.001)$ & $0.088(0.002)$ & $0.059(0.0035)$ \\
\hline 418 & $0.205(0.0023)$ & $0.180(0.0025)$ & $0.123(0.0026)$ & $0.158(0.0041)$ & $0.315(0.002)$ & $0.233(0.003)$ & $0.358(0.002)$ & $0.216(0.0035)$ \\
\hline 544 & $0.024(0.0022)$ & $0.022(0.0015)$ & $0.015(0.0015)$ & $0.019(0.0026)$ & $0.036(0.001)$ & $0.027(0.002)$ & $0.041(0.003)$ & $0.026(0.0025)$ \\
\hline 578 & $0.024(0.0014)$ & $0.022(0.0030)$ & $0.015(0.0021)$ & $0.019(0.002)$ & $0.036(0.001)$ & $0.027(0.002)$ & $0.041(0.002)$ & $0.026(0.015)$ \\
\hline \multirow{2}{*}{$\begin{array}{c}\lambda \\
(\mathbf{n m})\end{array}$} & \multicolumn{2}{|c|}{ Control (1\%) } & \multicolumn{2}{|c|}{ Experimental (1\%) } & \multicolumn{2}{|c|}{ Control (0.5\%) } & \multicolumn{2}{|c|}{ Experimental $(0.5 \%)$} \\
\hline & 0 min & $30 \mathrm{~min}$ & 0 min & $30 \mathrm{~min}$ & 0 min & $30 \mathrm{~min}$ & 0 min & $30 \mathrm{~min}$ \\
\hline 280 & $0.257(0.0015)$ & $0.257(0.0020)$ & $0.275(0.0025)$ & $0.247(0.0015)$ & $0.398(0.001)$ & $0.395(0.003)$ & $0.347(0.001)$ & $0.359(0.004)$ \\
\hline 346 & $0.040(0.0012)$ & $0.043(0.0015)$ & $0.052(0.001)$ & $0.037(0.002)$ & $0.061(0.001)$ & $0.060(0.002)$ & $0.034(0.002)$ & $0.04(0.0015)$ \\
\hline 418 & $0.120(0.0011)$ & $0.162(0.0015)$ & $0.171(0.002)$ & $0.133(0.0026)$ & $0.286(0.001)$ & $0.278(0.003)$ & $0.124(0.001)$ & $0.1565(0.0035)$ \\
\hline 544 & $0.020(0.0014)$ & $0.020(0.001)$ & $0.026(0.0015)$ & $0.017(0.0015)$ & $0.022(0.001)$ & $0.022(0.002)$ & $0.014(0.014)$ & $0.018(0.0025)$ \\
\hline 578 & $0.020(0.0007)$ & $0.020(0.0025)$ & $0.026(0.0015)$ & $0.017(0.001)$ & $0.022(0.002)$ & $0.022(0.003)$ & $0.014(0.002)$ & $0.018(0.004)$ \\
\hline
\end{tabular}

The changes in the optical density were significant with some doses of Arsenicum album and there were significant differences in the optical densities after 30 minutes and 60 minutes of incubation at some wavelengths. In chicken blood, the OD values decreased significantly at $418 \mathrm{~nm}$ with $4 \%, 2 \%, 1.34 \%$ and $1 \%$ experimental samples after 30 minutes of incubation; at $280 \mathrm{~nm}$ and $346 \mathrm{~nm}$, the OD values decreased with $1.34 \%$ and $1 \%$ experimental samples after 30 minutes of incubation. It was also observed that the OD values decreased significantly at all the five wavelengths considered with $1.34 \%$ experimental sample (Figure 1).

In goat blood, the OD values decreased significantly at $280 \mathrm{~nm}$ with $2 \%$ and $1 \%$ experimental samples after 30 minutes and 60 minutes of incubation and little bit at $346 \mathrm{~nm}$ with $1 \%$ experimental sample. At $1.34 \%$, the medicine increased the OD values at all wavelengths studied while in control, the OD values were found to be decreased (Figure 2). 
Table 2: Changes in the optical density of blood after incubation with Arsenicum album of various strengths with goat blood at selected wavelengths at three time intervals viz. just after incubation, 30 minutes after incubation and 60 minutes after incubation [Values are expressed as Mean(SD)]

\begin{tabular}{|c|c|c|c|c|c|c|}
\hline \multirow{2}{*}{$\begin{array}{c}\lambda \\
(\mathrm{nm})\end{array}$} & \multicolumn{3}{|c|}{ Control (2\%) } & \multicolumn{3}{|c|}{ Experimental (2\%) } \\
\hline & $0 \mathrm{~min}$ & $30 \mathrm{~min}$ & $60 \mathrm{~min}$ & $0 \mathrm{~min}$ & $30 \mathrm{~min}$ & $60 \mathrm{~min}$ \\
\hline 280 & $0.560(0.0025)$ & $0.562(0.0025)$ & $0.504(0.003)$ & $0.554(0.0030)$ & $0.514(0.0035)$ & $0.52(0.025)$ \\
\hline 346 & $0.016(0.002)$ & $0.017(0.002)$ & $0.017(0.0015)$ & $0.014(0.002)$ & $0.023(0.002)$ & $0.024(0.0025)$ \\
\hline 418 & $0.051(0.0025)$ & $0.172(0.002)$ & $0.054(0.0025)$ & $0.044(0.0025)$ & $0.042(0.0015)$ & $0.057(0.0035)$ \\
\hline 544 & $0.005(0.002)$ & $0.019(0.003)$ & $0.006(0.0025)$ & $0.004(0.0002)$ & $0.008(0.0015)$ & $0.01(0.0025)$ \\
\hline 578 & $0.005(0.003)$ & $0.019(0.0045)$ & $0.006(0.0015)$ & $0.004(0.00015)$ & $0.008(0.0025)$ & $0.01(0.002)$ \\
\hline \multirow{2}{*}{$\begin{array}{c}\lambda \\
(\mathbf{n m})\end{array}$} & \multicolumn{3}{|c|}{ Control $(1.34 \%)$} & \multicolumn{3}{|c|}{ Experimental (1.34\%) } \\
\hline & 0 min & $30 \mathrm{~min}$ & $60 \mathrm{~min}$ & 0 min & $30 \mathrm{~min}$ & $60 \mathrm{~min}$ \\
\hline 280 & $0.551(0.002)$ & $0.517(0.0045)$ & $0.564(0.0035)$ & $0.525(0.0035)$ & $0.545(0.0035)$ & $0.525(0.0035)$ \\
\hline 346 & $0.029(0.003)$ & $0.013(0.002)$ & $0.033(0.0035)$ & $0.016(0.0035)$ & $0.03(0.0025)$ & $0.024(0.0045)$ \\
\hline 418 & $0.078(0.0025)$ & $0.043(0.0025)$ & $0.137(0.0015)$ & $0.037(0.0015)$ & $0.072(0.002)$ & $0.099(0.0025)$ \\
\hline 544 & $0.011(0.0025)$ & $0.003(0.0003)$ & $0.015(0.002)$ & $0.003(0.0012)$ & $0.013(0.002)$ & $0.01(0.0004)$ \\
\hline 578 & $0.011(0.0015)$ & $0.003(0.0003)$ & $0.015(0.0025)$ & $0.003(0.00064)$ & $0.013(0.0035)$ & $0.01(0.0002)$ \\
\hline \multirow{2}{*}{$\begin{array}{c}\lambda \\
(\mathrm{nm})\end{array}$} & \multicolumn{3}{|c|}{ Control (1\%) } & \multicolumn{3}{|c|}{ Experimental (1\%) } \\
\hline & 0 min & $30 \mathrm{~min}$ & $60 \mathrm{~min}$ & 0 min & $30 \mathrm{~min}$ & $60 \mathrm{~min}$ \\
\hline 280 & $0.416(0.0035)$ & $0.412(0.0030)$ & $0.464(0.0035)$ & $0.474(0.0045)$ & $0.429(0.0055)$ & $0.459(0.0035)$ \\
\hline 346 & $0.020(0.0015)$ & $0.018(0.0035)$ & $0.054(0.0045)$ & $0.032(0.0025)$ & $0.03(0.0025)$ & $0.029(0.0015)$ \\
\hline 418 & $0.050(0.0035)$ & $0.114(0.0045)$ & $0.099(0.0045)$ & $0.077(0.005)$ & $0.102(0.0035)$ & $0.074(0.0025)$ \\
\hline 544 & $0.007(0.0015)$ & $0.013(0.0035)$ & $0.020(0.0035)$ & $0.01(0.0015)$ & $0.012(0.0035)$ & $0.009(0.0011)$ \\
\hline 578 & $0.007(0.003)$ & $0.013(0.0025)$ & $0.020(0.003)$ & $0.01(0.0015)$ & $0.012(0.003)$ & $0.009(0.0010)$ \\
\hline \multirow{2}{*}{$\begin{array}{c}\lambda \\
(\mathbf{n m})\end{array}$} & \multicolumn{3}{|c|}{ Control (0.5\%) } & \multicolumn{3}{|c|}{ Experimental $(0.5 \%)$} \\
\hline & 0 min & $30 \mathrm{~min}$ & $60 \mathrm{~min}$ & 0 min & $30 \mathrm{~min}$ & $60 \mathrm{~min}$ \\
\hline 280 & $0.388(0.0025)$ & $0.398(0.0025)$ & $0.399(0.004)$ & $0.387(0.0015)$ & $0.399(0.0035)$ & $0.403(0.003)$ \\
\hline 346 & $0.031(0.002)$ & $0.030(0.0025)$ & $0.030(0.003)$ & $0.029(0.004)$ & $0.036(0.0025)$ & $0.032(0.003)$ \\
\hline 418 & $0.094(0.0025)$ & $0.096(0.002)$ & $0.100(0.00208)$ & $0.091(0.002)$ & $0.104(0.0015)$ & $0.104(0.0025)$ \\
\hline 544 & $0.013(0.0025)$ & $0.014(0.003)$ & $0.014(0.0025)$ & $0.012(0.001)$ & $0.017(0.002)$ & $0.015(0.0025)$ \\
\hline 578 & $0.013(0.0035)$ & $0.014(0.004)$ & $0.014(0.0005)$ & $0.012(0.0011)$ & $0.017(0.0005)$ & $0.015(0.0015)$ \\
\hline
\end{tabular}

\section{DISCUSSION}

The medicinal plant leaf extracts have been known to influence the morphology of human erythrocytes (Yadav et al., 2018). They also influence the biochemical parameters of blood in vitro (Yadav et al., 2020). Actually, the changes in the constituents of the serum tell us a lot 
about the health of the person and spectroscopy is a convenient way of knowing the 'serum health'.

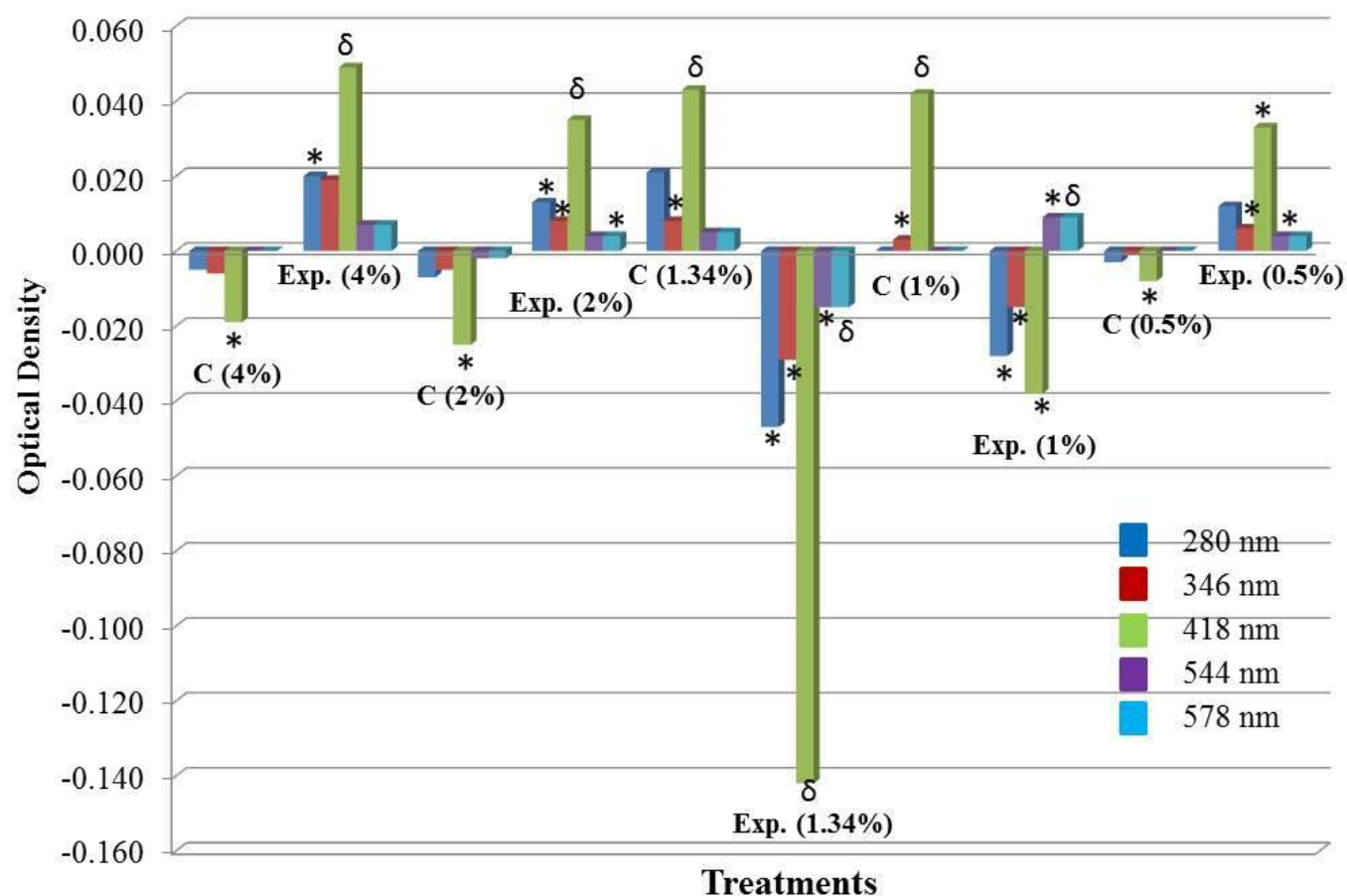

Figure 1: Difference in the OD values of chicken blood 30 minutes after incubation with Arsenicum album $(*=\mathrm{p} \leq 0.05 ; \delta=\mathrm{p} \leq 0.001)$

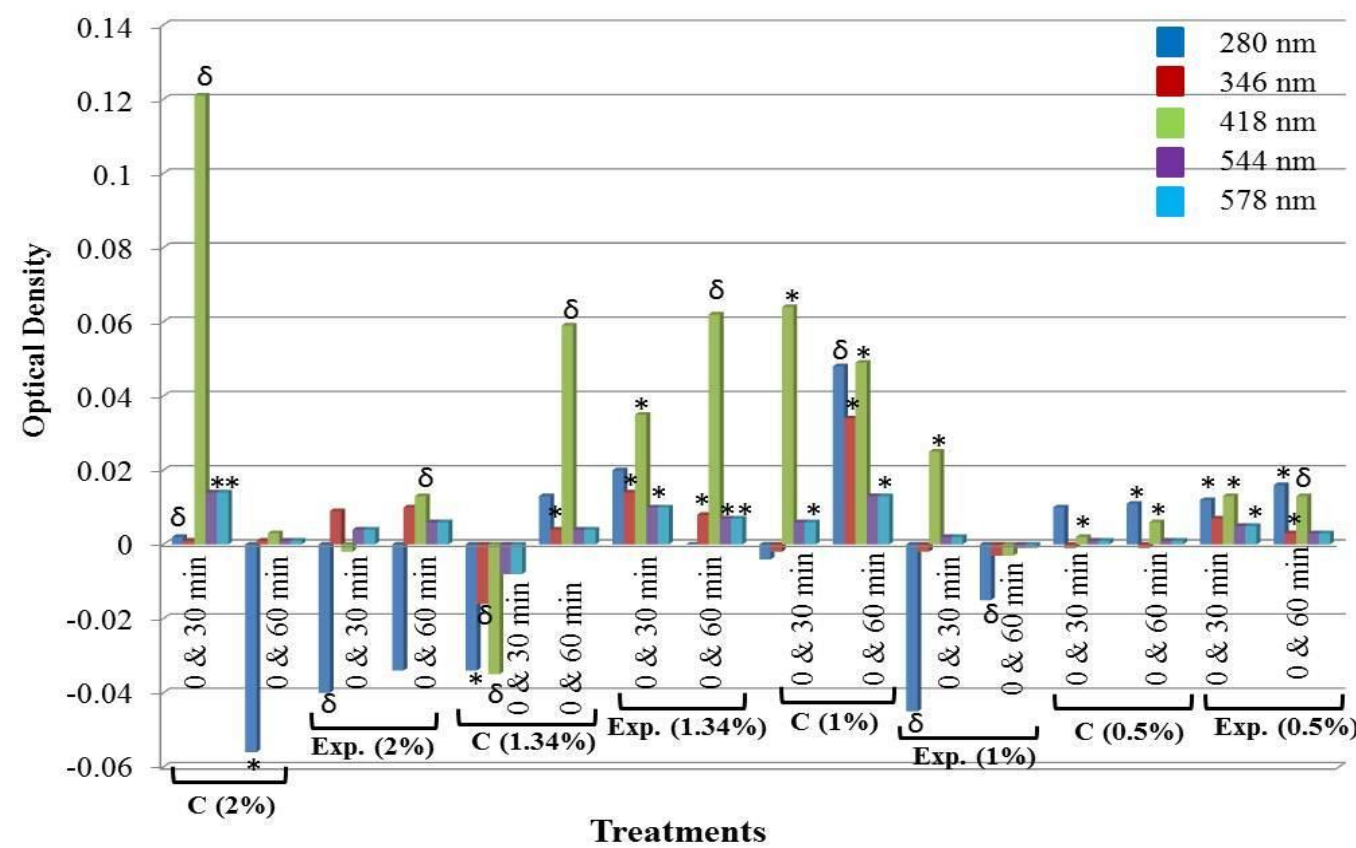

Figure 2: Difference in the OD values of goat blood with passage of time i.e. 30 minutes and 60 minutes after incubation with Arsenicum album $(*=\mathrm{p} \leq 0.05 ; \delta=\mathrm{p} \leq 0.001)$ 
The levels of protein, amino acids, glucose metabolism status and carboxyhemoglobin/oxyhemoglobin can be measured directly using UV-visible spectroscope (Srinivasan et al., 2004). Further, Fourier Transform InfraRed (FTIR) spectroscopy is an established biodiagnostics tool for early detection and diagnosis of diabetes (Gunasekaran $e t$ $a l ., 2008$ ). The InfraRed (IR) and FTIR spectroscopy can be used to study the changes in the serum components which may be diagnostic of diseases like diabetes.

In our study, after incubation of chicken blood with selected doses of Arsenicum album, it was observed the OD values increased significantly at $280 \mathrm{~nm}(\mathrm{p} \leq 0.05)$ and $418 \mathrm{~nm}(\mathrm{p} \leq 0.001)$ for $4 \% ; 280 \mathrm{~nm}(\mathrm{p} \leq 0.05), 346 \mathrm{~nm}(\mathrm{p} \leq 0.05), 418 \mathrm{~nm}(\mathrm{p} \leq 0.001)$ and $578 \mathrm{~nm}(\mathrm{p} \leq 0.05)$ for $2 \%$ and $346 \mathrm{~nm}(\mathrm{p} \leq 0.05), 418 \mathrm{~nm}(\mathrm{p} \leq 0.05)$ and $544 \mathrm{~nm}(\mathrm{p} \leq 0.05)$ for $0.5 \%$ dose of Arsenicum album as compared to control. However, after incubation with $1.34 \%$ Arsenicum album the OD values decreased significantly at $280 \mathrm{~nm}, 346 \mathrm{~nm}, 418 \mathrm{~nm}, 544 \mathrm{~nm}$ and $548 \mathrm{~nm}$ after 30 minutes of incubation while with $1 \%$ medicine, the OD values decreased at $280 \mathrm{~nm}, 346 \mathrm{~nm}$ and $418 \mathrm{~nm}$. The significant $(\mathrm{p} \leq 0.05)$ decrease in OD value at $280 \mathrm{~nm}$ suggests that there was low amino acid level which means less protein breakdown and less cellular breakdown. The decrease in OD values at $346 \mathrm{~nm}(\mathrm{p} \leq 0.05)$ suggests that the levels of NADH and NADPH were lowered after 30 minutes of incubation with $1.34 \%$ and $1 \%$ Arsenicum album which implies that there was reduction in the carbohydrate metabolism (Yadav et al., 2020) and it also indicates a reduction in the oxidative stress(Fernandez-Marcos \& Nobrega-Pereira, 2016). The low OD value at $418 \mathrm{~nm}$ in $1.34 \%(\mathrm{p} \leq 0.001)$ and $1 \%(\mathrm{p} \leq 0.05)$ dose of Arsenicum album suggests that there was low heme breakdown in the erythrocytes. However, the most striking reduction in the OD values after 30 minutes of incubation was observed at $418 \mathrm{~nm}$ in $1.34 \%$ Arsenicum album incubation suggesting that the constituents of medicine played crucial role in significantly preventing the heme catabolism. The levels of carboxyhemoglobin and oxyhemoglobin were significantly $(\mathrm{p} \leq 0.05)$ lowered only in $1.34 \%$ experimental samples suggesting that there was less production of endogenous carbon monoxide (CO). The endogenous source of CO is haemoglobin degradation (Setiawan et al., 2020) catalysed by hemeoxygenase (McArdle et al., 2016), so the reduction in OD at $418 \mathrm{~nm}$ suggests that there was less hemedegradation and less breakdown of erythrocytes. Moreover, since the levels of carboxyheoglobin and oxyhemoglobin can't be distinguished by wavelengths, hence we measure their levels at $544 \mathrm{~nm}$ and $578 \mathrm{~nm}$ together (Blumenthal, 2001). Thus, the therapeutic role of Arsenicum album on chicken blood was observed maximum with $1.34 \%$ medicine and it reduced with $1 \%$ medicine after 30 minutes of incubation. 
When the goat blood was incubated with various strengths $(4 \%, 2 \%, 1.34 \%, 1 \%$ and $0.5 \%)$ of Arsenicum album, the OD values did show some variation as compared to control. In experimental sample with $2 \%$ medicine, the OD values decreased significantly after 30 minutes of incubation at $280 \mathrm{~nm}(\mathrm{p} \leq 0.05)$. This indicates less protein breakdown suggesting less cellular damage. The same was observed after 60 minutes of incubation. Similarly, with $1 \%$ medicine incubation, the OD value decreased at $280 \mathrm{~nm}$ after 30 minutes of incubation $(\mathrm{p} \leq 0.001)$ as well as after 60 minutes $(\mathrm{p} \leq 0.001)$ of incubation suggesting a therapeutic role of medicine. However, as time passed i.e. from 30 minutes to 60 minutes of incubation, the difference in OD values was found to be lowered suggesting that the medicine was more effective in protecting integrity of erythrocytes after 30 minutes of incubation as against after 60 minutes of incubation at $2 \%$ and $1 \%$ strengths. It is interesting to note here that in $2 \%$ control the OD value was very high at $418 \mathrm{~nm}(\mathrm{p} \leq 0.001)$ suggesting huge haemoglobin breakdown, however after incubation with $2 \%$ medicine, the OD values decreased. This clearly suggests a positive role of medicine in maintaining integrity of erythrocytes.

The experimental samples of goat blood at $4 \%, 1.34 \%$ and $0.5 \%$ showed an increase in the OD values after 30 minutes $(\mathrm{p} \leq 0.05)$ and 60 minutes $(\mathrm{p} \leq 0.05)$ of incubation. This suggests that these doses of Arsenicum album led to an increase in cellular breakdown, increase in carbohydrate metabolism and oxidative stress as well as higher levels of carboxyhemoglobin and oxyhemoglobin indicating more endogenous production of $\mathrm{CO}$ in the erythrocytes. All these observations lead us to the conclusion that these three doses of Arsenicum album had more deleterious effects on the cellular components of goat blood, especially erythrocytes. Thus, of the five doses considered in the study, only $2 \%$ and $1 \%$ doses of Arsenicum album showed some therapeutic influence, especially with respect to protein breakdown, on goat blood after 30 minutes and 60 minutes of incubation.

\section{CONCLUSION}

From our results, it can be concluded that in chicken blood, 1.34\% Arsenicum album showed maximum therapeutic effects as it lowered protein breakdown, lowered carbohydrate metabolism and oxidative stress and also lowered the levels of carboxyhemoglobin and oxyhemoglobin after 30 minutes of incubation. With 1\% Arsenicum album, there was reduced cellular breakdown, lowered carbohydrate metabolism and hemoglobin degradation after 30 minutes of incubation. In goat blood, Arsenicum album showed healing effects only with two doses i.e. $2 \%$ and $1 \%$. After incubation with $2 \%$ and $1 \%$ Arsenicum album, the cellular breakdown was significantly lowered after 30 minutes and 60 minutes of incubation while the 
other doses of Arsenicum album did not show any therapeutic effects on the cellular components of blood and in fact induced deleterious effects.

\section{CONFLICT OF INTEREST}

None

\section{SOURCE OF FUNDING}

Authors are thankful to Maitreyi College, University of Delhi for necessary financial aid for the work under its Summer Internship Program.

\section{ACKNOWLEDGEMENT}

Authors thank the Principal, Maitreyi College, University of Delhi for her continuous support and encouragement.

\section{REFERENCES}

Antonelli, M. \& Donelli, D. (2019). Reinterpreting homeopathy in the light of placebo effects to manage patients who seek homeopathic care: A systematic review. Health and Social Care in Community, 27(4), 824-847. https://doi.org/10.1111/hsc.12681

Beghi, G.M. \& Morselli-Labate, A.M. (2016). Does homeopathic medicine have a preventive effect on respiratory tract infections? A real life observational study. Multidisciplinary respiratory medicine, 11, 12. https://doi.org/10.1186/s40248-016-0049-0

Blumenthal, I. (2001). Carbon monoxide poisoning. Journal of the Royal Society of Medicine, 94(6), 270-272. https://dx.doi.org/10.1177\%2F014107680109400604

Caulfield, T. \& DeBow, S. (2005). A systematic review of how homeopathy is represented in conventional and CAM peer reviewed journals. BMC complementary and alternative medicine; 5,12. https://doi.org/10.1186/1472-6882-5-12

Fernandez-Marcos, P.J. \& Nobrega-Pereira, S. (2016). NADPH: new oxygen for the ROS theory of aging. Oncotarget, $7 \quad$ (32), 50814-50815. https://doi.org/10.18632/oncotarget.10744

Gunasekaran, S., Natarajan, R.K., Renganayaki, V. \& Rathikha, R. (2008). FTIR and UV Visible Spectrophotometric Approach to Discriminate Leukemic Sera. Asian Journal of Chemistry, 20 (4), 2521-2530. 
Ive, E.C., Couchman, I.M.S. \& Reddy, L. (2012). Therapeutic effect of Arsenicum album on leukocytes. International journal of molecular sciences, 13(3), 3979-3987. https://doi.org/10.3390/ijms13033979

Khuda-Baksh, A.R., Roy-Karmakar, S., Banerjee, A., Banerjee, P., Pathak, S., Biswas, S.J., Haque, S., Das, D., Boujedaini, N. \& Belon, P. (2011). A follow-up study on the efficacy of the homeopathic remedy Arsenicum album in volunteers living in high risk arsenic contaminated areas. Evidence-based complementary and alternative medicine :eCAM, 11: Article ID 129214. https://doi.org/10.1093/ecam/nep122

Louden, I. (2006). A brief history of homeopathy. Journal of the Royal Society of Medicine, 99(12), 607-610. https://dx.doi.org/10.1258\%2Fjrsm.99.12.607

McArdle, A.J., Webbe, J., Sim, K., Parrish, G., Hoggart, C., Wang, Y., Kroll, J.S., Godambe, S. \& Cunnington, A.J. (2016). Determinants of carboxyhemoglobin levels and relationship with sepsis in a retrospective cohort of preterm neonates. PLOS ONE, https://doi.org/10.1371/journal.pone.0161784

Setiawan, D., Nurmalasari, A., Farihatun, A., Aulia, R.S. \& Noviani, I. (2020). A relationship between a level of carboxyhemoglobin and haemoglobin in bus mechanics. Journal of Physics: Conference Series, 1477 (6):062012. https://doi.org/10.1088/1742$\underline{6596 / 1477 / 6 / 062012}$

Srinivasan, S., Ponnambalam, U. \& Gunasekaran, S. (2004). Measurement of optical density of blood with varying time using UV-Vis spectroscopic method. Asian Journal of Chemistry, 16(1), 23-26.

Steuter, E. (2002). Contesting the rule(s) of medicine: homeopathy's battle for legitimacy. Journal of Canadian Studies (Special Issue on Science and Politics in Canada.), 37 (3), 92-111. https://doi.org/10.3138/jcs.37.3.92

Vilsveen, P., Fibert, P. \& Relton, C. (2018). Homeopathy in the treatment of depression: a systematic review. European Journal of Integrative Medicine, 22, 22-36. https://doi.org/10.1016/j.eujim.2018.07.004.

Yadav, M., Chaturvedi G. \& Jha, N. (2018). Morphological changes in human RBCs due to exposure to selected medicinal plant leaf extracts in vitro. International Journal of Pharmaceutical Sciences and Research, 9, 2405-2410. doi:10.13040/IJPSR.09758232.9(6).2405-10.

Yadav, M., Chaturvedi, G. \& Jha, N. (2020). Effect of leaf extracts of selected medicinal plants on blood against hypotonic solution by measuring optical density: An in vitro study. 
Indian Journal of Experimental Biology, 58(6), 420-425. http://nopr.niscair.res.in/handle/123456789/54446

How to cite this article: Yadav, M., Yadav, M. \& Ranjan. L. (2020). Influence of the homeopathic medicine, Arsenicum album, on selected biochemical parameters of avian and mammalian blood in vitro. Vantage: Journal of Thematic Analysis, 1(1), 136-146.

DOI: https://doi.org/10.52253/vjta.2020.v01i01.12

(C) The Author(s) 2020.

This work is licensed under a Creative Commons Attribution 4.0 International License which permits its use, distribution and reproduction in any medium, provided the original work is cited. 